\title{
Comparative Study of Three Achillea Essential Oils from Eastern Part of Turkey and their Biological Activities
}

\author{
Betül Demirci $^{\oplus 1^{*}}$, K. Hüsnü Can Başer ${ }^{\oplus 2}$, Zeki Aytaç ${ }^{\oplus 3}$, Shabana I. Khan ${ }^{\oplus 4}$, \\ Melissa R. Jacob ${ }^{\oplus 4}$ and Nurhayat Tabanca ${ }^{\oplus 4,5}$
}

\author{
${ }^{I}$ Department of Pharmacognosy, Faculty of Pharmacy, Anadolu University, 26470, Eskisehir, Türkiye \\ ${ }^{2}$ Near East University, Faculty of Pharmacy, Department of Pharmacognosy, Lefkoşa (Nicosia), N. Cyprus \\ ${ }^{3}$ Department of Biology, Faculty of Science, Gazi University, 06500 Ankara, Türkiye \\ ${ }^{4}$ National Center for Natural Products Research, The University of Mississippi, University, MS 38677, USA \\ ${ }^{5}$ USDA-ARS, Subtropical Horticulture Research Station, 13601 Old Cutler Rd., Miami, FL 33158 USA
}

(Received March 30, 2017; Revised July 24, 2017; Accepted July 26, 2017)

\begin{abstract}
Essential oils obtained by hydrodistillation were analyzed both by gas chromatography (GC) and gas chromatography-mass spectrometry (GC-MS). The main constituents found in Achillea oil were as follows: $A$. filipendulina Lam.: 43.8\% santolina alcohol, $14.5 \%$ 1,8-cineole and $12.5 \%$ cis-chrysanthenyl acetate; A. magnifica Hiemerl ex Hub.-Mor.: 27.5\% linalool, 5.8\% spathulenol, 5.5\% terpinen-4-ol, 4.7\% $\alpha$-terpineol and $4.7 \% \beta$-eudesmol; A. tenuifolia Lam.: 12.4\% artemisia ketone, 9.9\% p-cymene, 7.1\% camphor, 5.9\% terpinen-4-ol, 4.7\% caryophyllene oxide and $4.5 \% \alpha$-pinene. Furthermore, the Achillea essential oils were evaluated for antimalarial and antimicrobial activities. A. magnifica and A. filipendulina oils showed strong antimalarial activity against both chloroquine sensitive D6 $\left(\mathrm{IC}_{50}=1.2\right.$ and $\left.0.68 \mu \mathrm{g} / \mathrm{mL}\right)$ and chloroquine resistant $\mathrm{W} 2\left(\mathrm{IC}_{50}=1.1\right.$ and $\left.0.9 \mu \mathrm{g} / \mathrm{mL}\right)$ strains of Plasmodium falciparum without any cytotoxicity to mammalian cells up to $\mathrm{IC}_{50}=47.6 \mu \mathrm{g} / \mathrm{mL}$ against Vero cells. whereas $A$. tenuifolia oil showed no antimalarial activity up to a concentration of $20 \mathrm{mg} / \mathrm{mL}$. All three Achillea oils showed no antibacterial activity against human pathogenic bacteria up to a concentration of $200 \mu \mathrm{g} / \mathrm{mL}$. A. tenuifolia and A. magnifica oils demonstrated mild antifungal activity against Cryptococcus neoformans $\left(\mathrm{IC}_{50}=45,20\right.$ and $15 \mu \mathrm{g} / \mathrm{mL}$, respectively).
\end{abstract}

Keywords: Asteraceae; Achillea filipendulina; A. magnifica; A. tenuifolia; essential oil composition; antimalarial and antimicrobial. (C) 2018 ACG Publications. All rights reserved.

\section{Plant Source}

Achillea $\mathrm{L}$. is a large genus belonging to the family Asteraceae. This genus is widely distributed in Anatolia and is represented by 59 species of which 31 are endemic for Turkey [1]. Achillea species comprise an important biological resource in folk medicine to treat various diseases and several of them are used for their pharmaceutical, cosmetic, and fragrance properties [3]. Achillea species are generally known as "Civanperçemi" and used for the treatment of gastrointestinal disorders in Anatolian folk medicine. In the present study, we investigated three Achillea species (A. filipendulina Lam., A. tenuifolia Lam., and A.

\footnotetext{
${ }^{*}$ Corresponding author: E-Mail: betuldemirci@gmail.com; Phone:+90 22233505 80-3717
} 
magnifica Hiemerl ex Hub.-Mor. from eastern part of Turkey. Out of the three species, A. magnifica, an endemic species, is recognized by local names as "karcivanı" and its dried flowering parts are mixed with honey and consumed for stomach disorders [4].

The plant material was identified by Prof. Dr. Z. Aytaç. Voucher specimens were deposited at the Herbarium of Gazi University, Faculty of Science and Letters. Detailed information on the plant materials used are given in Table 1.

\section{Previous Studies}

Essential oils of Achillea species have been the subject of several investigations. A. millefolium L. has been the most widely studied species because of its economic value and therapeutic properties. Previous phytochemical investigations of Achillea species have revealed the presence of sesquiterpene lactones, proazulenes, sesquiterpenoids, flavonoids, triterpenes, coumarins, peroxides, phenolic and polyacetylene compounds [5].

Although the essential oil composition of A. filipendulina, A. tenuifolia and A. magnifica have been recently reported in the literature and the antibacterial activity of $A$. filipendulina has been studied [5-10], the antimalarial activity of A. tenuifolia and antimicrobial activity of A. tenuifolia and A. magnifica were investigated for the first time.

\section{Present Studies}

Isolation of the Essential Oil: Essential oils were hydrodistilled from dried aerial parts for $3 \mathrm{~h}$ using a Clevenger apparatus. The yields were calculated on a dry weight basis as given in Table 1 .

GC-MS and GC-FID Analysis: The GC-MS and GC-FID analysis were carried out with an Agilent 5975 GC-MSD and Agilent 6890N GC systems, respectively. Analysis conditions and identification of the oil components are similar to our earlier studies [1].

Biological Activity: The in vitro antimalarial [11] and antimicrobial [12] activity was performed as previously described. Antimalarial standards chloroquine (Aldrich-Sigma, ST, Louis, MO) and artemisinin (Aldrich-Sigma, ST, Louis, MO) and antimicrobial standards ciprofloxacin (ICN Biomedicals, Ohio) for bacteria and amphotericin B (ICN Biomedicals, Ohio) for fungi were purchased from commercial sources.

Table 1. Information on the plant material and essential oils

\begin{tabular}{|c|c|c|c|c|c|}
\hline Achillea sp. & Collection site & $\begin{array}{l}\text { Altitude } \\
\text { (m) }\end{array}$ & $\begin{array}{c}\text { Collection } \\
\text { period }\end{array}$ & $\begin{array}{c}\text { Oil yield }^{1} \\
(\%)\end{array}$ & $\mathrm{ZA}^{2}$ \\
\hline A. filipendulina & $\begin{array}{l}\text { Şırnak-Şenova-Hakkari } 79 . \\
\text { km }\end{array}$ & 1950 & 15.07 .2001 & 0.80 & ZA8195 \\
\hline A. magnifica & Malatya: Pütürge, $17 . \mathrm{km}$ & 1100 & 12.07 .2001 & 0.09 & ZA 8135 \\
\hline A. tenuifolia & $\begin{array}{l}\text { Ağrı-Doğubeyazıt-Igdır, } 13 \text {. } \\
\text { km }\end{array}$ & 1600 & 19.07.2001 & 0.20 & ZA8272 \\
\hline
\end{tabular}

Air-dried aerial parts of three Achillea species, A. filipendulina Lam., A. magnifica Hiemerl ex Hub.Mor., and A. tenuifolia Lam., were analyzed by GC and GC-MS and the individual identified components with their relative percentages are given in Table 2.

In the oil of the A. filipendulina, 53 components were characterized representing $93 \%$ of the total oil. This oil was characterized by a relatively high content of santolina alcohol (43.8\%). 1,8-Cineole (14.5\%) and cis-chrysanthenyl acetate (12.5\%) were found as other main constituents. 
A total of 70 compounds were characterized in A. magnifica essential oil, representing $91.7 \%$ of the total oil with linalool (27.5\%), spathulenol (5.8\%), terpinen-4-ol (5.5\%), $\alpha$-terpineol (4.7\%) and $\beta$-eudesmol $(4.7 \%)$ as main constituents.

Table 2. The composition of the essential oils of three Achillea species

\begin{tabular}{|c|c|c|c|c|c|c|}
\hline RRI $^{\mathbf{a}}$ & $\mathbf{R R I}^{\mathbf{b}}$ & Compound & Af $(\%)^{c}$ & $\operatorname{Am}(\%)^{c}$ & At $(\%)^{c}$ & $I^{M^{d}}$ \\
\hline $1025^{f}$ & 1032 & $\alpha$-Pinene & 1.3 & 0.1 & 4.5 & $\mathrm{tR}, \mathrm{MS}$ \\
\hline $1036^{\mathrm{g}}$ & 1043 & Santolinatriene & 2.3 & - & 3.6 & MS \\
\hline $1068^{g}$ & 1076 & Camphene & 0.9 & - & 1.6 & $\mathrm{tR}, \mathrm{MS}$ \\
\hline $1082^{\mathrm{g}}$ & 1093 & Hexanal & - & - & 0.3 & $\mathrm{tR}, \mathrm{MS}$ \\
\hline $1117^{\mathrm{f}}$ & 1118 & $\beta$-Pinene & 0.9 & 0.1 & 1.2 & $\mathrm{tR}, \mathrm{MS}$ \\
\hline $1122^{\mathrm{f}}$ & 1132 & Sabinene & 0.2 & - & 0.4 & $\mathrm{tR}, \mathrm{MS}$ \\
\hline $1122^{\mathrm{g}}$ & 1135 & Thuja-2,4(10)-diene & - & - & 0.1 & MS \\
\hline \multirow[t]{2}{*}{$1160^{\mathrm{f}}$} & 1174 & Myrcene & - & - & 0.3 & $\mathrm{tR}, \mathrm{MS}$ \\
\hline & 1185 & Isobutyl 2-methyl butyrate & - & - & 0.1 & MS \\
\hline $1192^{\mathrm{g}}$ & 1195 & Dehydro-1,8-cineole & 0.2 & - & 0.1 & MS \\
\hline $1212^{\mathrm{f}}$ & 1203 & Limonene & - & 0.3 & 0.6 & $\mathrm{tR}, \mathrm{MS}$ \\
\hline $1211^{\mathrm{g}}$ & 1213 & 1,8-Cineole & 14.5 & 1.9 & 2.3 & $\mathrm{tR}, \mathrm{MS}$ \\
\hline $1232^{\mathrm{f}}$ & 1244 & 2-Pentyl furan & - & - & 0.1 & MS \\
\hline $1245^{\mathrm{f}}$ & 1255 & $\gamma$-Terpinene & - & - & 0.2 & $\mathrm{tR}, \mathrm{MS}$ \\
\hline \multirow[t]{2}{*}{$1281^{\mathrm{f}}$} & 1280 & $p$-Cymene & 1.5 & 0.4 & 9.9 & $\mathrm{tR}, \mathrm{MS}$ \\
\hline & 1285 & Isoamyl isovalerate & 0.1 & - & - & MS \\
\hline \multirow[t]{5}{*}{$1282^{f}$} & 1290 & Terpinolene & - & - & 0.1 & $\mathrm{tR}, \mathrm{MS}$ \\
\hline & 1296 & Pentyl isovalerate & - & 0.1 & - & MS \\
\hline & 1355 & 1,2,3-Trimethyl benzene & 0.1 & - & - & MS \\
\hline & 1358 & Artemisia ketone & - & - & 12.4 & MS \\
\hline & 1386 & Octenyl acetate & - & - & 0.2 & MS \\
\hline \multirow[t]{4}{*}{$1395^{\mathrm{g}}$} & 1403 & Yomogi alcohol & 0.5 & - & 2.0 & MS \\
\hline & 1405 & Santolina alcohol & 43.8 & - & 0.1 & MS \\
\hline & 1429 & Artemisyl acetate & 0.2 & - & 0.2 & MS \\
\hline & 1431 & $7 \alpha-(H)-S i l p h i p e r f o l-5-e n e$ & - & - & 0.3 & MS \\
\hline \multirow[t]{2}{*}{$1446^{\mathrm{g}}$} & 1450 & trans-Linalool oxide (Furanoid) & - & 1.0 & - & MS \\
\hline & 1454 & $7 \beta$-(H)-silphiperfol-5-ene & - & - & 0.1 & MS \\
\hline $1548^{g}$ & 1474 & trans-Sabinene hydrate & 0.2 & 2.3 & 1.8 & MS \\
\hline \multirow[t]{3}{*}{$1454^{\mathrm{g}}$} & 1478 & cis-Linalool oxide (Furanoid) & - & 0.8 & - & MS \\
\hline & 1478 & Linalool-7-oxide-3-one & - & 0.2 & - & MS \\
\hline & 1487 & Isoneroloxide-I & - & - & 0.3 & MS \\
\hline \multirow[t]{3}{*}{$1458^{f}$} & 1497 & $\alpha$-Copaene & - & $\mathrm{t}^{\mathrm{e}}$ & 0.1 & MS \\
\hline & 1499 & $\alpha$-Campholene aldehyde & 0.1 & $\mathrm{t}$ & 0.1 & MS \\
\hline & 1501 & Silphiperfol-6-ene & - & - & $\operatorname{tr}$ & MS \\
\hline $1510^{\mathrm{g}}$ & 1510 & Artemisia alcohol & 0.3 & - & 3.6 & MS \\
\hline $1515^{\mathrm{h}}$ & 1532 & Camphor & 0.6 & 1.3 & 7.1 & $\mathrm{tR}, \mathrm{MS}$ \\
\hline $1518^{\mathrm{g}}$ & 1541 & Benzaldehyde & - & - & 0.3 & $\mathrm{tR}, \mathrm{MS}$ \\
\hline \multirow[t]{3}{*}{$1543^{\mathrm{h}}$} & 1553 & Linalool & 0.1 & 27.5 & 0.1 & $\mathrm{tR}, \mathrm{MS}$ \\
\hline & 1556 & cis-Sabinene hydrate & 0.2 & 1.1 & 1.0 & MS \\
\hline & 1562 & Isopinocamphone & 0.1 & - & - & MS \\
\hline $1584^{\mathrm{g}}$ & 1571 & trans-p-Menth-2-en-1-ol & 0.1 & 0.5 & 0.8 & MS \\
\hline $1561^{\mathrm{g}}$ & 1582 & cis-Chrysanthenyl acetate & 12.5 & - & - & MS \\
\hline $1575^{\mathrm{h}}$ & 1586 & Pinocarvone & - & 0.7 & 0.8 & $\mathrm{tR}, \mathrm{MS}$ \\
\hline $1579^{\mathrm{h}}$ & 1591 & Bornyl acetate & 1.4 & - & 0.1 & $\mathrm{tR}, \mathrm{MS}$ \\
\hline \multirow[t]{2}{*}{$1590^{\mathrm{h}}$} & 1600 & $\beta$-Elemene & - & - & 0.5 & MS \\
\hline & 1601 & Nopinone & - & 0.5 & - & MS \\
\hline $1601^{\mathrm{h}}$ & 1611 & Terpinen-4-ol & 0.8 & 5.5 & 5.9 & $\mathrm{tR}, \mathrm{MS}$ \\
\hline $1608^{\mathrm{h}}$ & 1612 & $\beta$-Caryophyllene & - & - & 0.2 & $\mathrm{tR}, \mathrm{MS}$ \\
\hline $1602^{\mathrm{h}}$ & 1616 & Hotrienol & - & 3.0 & - & MS \\
\hline $1614^{\mathrm{g}}$ & 1638 & cis- $p$-Menth-2-en-1-ol & 0.1 & 0.4 & 0.6 & MS \\
\hline $1631^{\mathrm{h}}$ & 1648 & Myrtenal & 0.1 & 1.4 & - & MS \\
\hline $1651^{\mathrm{h}}$ & 1651 & Sabinaketone & 0.2 & - & - & MS \\
\hline
\end{tabular}


Essential oil composition of Achillea species and their biological activities

\begin{tabular}{|c|c|c|c|c|c|c|}
\hline & 1655 & Isobornyl propionate & - & - & 0.1 & MS \\
\hline & 1656 & Chrysanthenyl isobutyrate & - & - & 0.2 & MS \\
\hline \multirow[t]{2}{*}{$1649^{\mathrm{g}}$} & 1661 & Alloaromadendrene & - & 0.1 & - & MS \\
\hline & & Table 2 Continued & & & & \\
\hline \multirow[t]{2}{*}{$1659^{\mathrm{g}}$} & 1663 & cis-Verbenol & 0.1 & - & 0.3 & $\mathrm{tR}, \mathrm{MS}$ \\
\hline & 1665 & cis-Sabinyl acetate & - & 0.1 & - & MS \\
\hline $1661^{\mathrm{h}}$ & 1670 & trans-Pinocarveol & 0.5 & 1.2 & 0.4 & $\mathrm{tR}, \mathrm{MS}$ \\
\hline \multirow[t]{2}{*}{$1679^{g}$} & 1682 & $\delta$-Terpineol & 0.1 & 0.3 & - & MS \\
\hline & 1682 & (E)-Ocimenol & - & - & 0.1 & MS \\
\hline $1680^{\mathrm{g}}$ & 1683 & trans-Verbenol & 0.3 & - & 1.6 & MS \\
\hline $1666^{\mathrm{g}}$ & 1687 & $\alpha$-Humulene & - & 0.1 & - & $\mathrm{tR}, \mathrm{MS}$ \\
\hline \multirow[t]{2}{*}{$1710^{\mathrm{g}}$} & 1689 & trans-piperitol & 0.1 & 0.2 & - & MS \\
\hline & 1688 & Selina-4,11-diene & - & 0.3 & - & MS \\
\hline $1694^{\mathrm{g}}$ & 1706 & $\alpha$-Terpineol & 0.2 & 4.7 & 0.8 & $\mathrm{tR}, \mathrm{MS}$ \\
\hline $1699^{\mathrm{g}}$ & 1719 & Borneol & 2.9 & 0.2 & 0.5 & $\mathrm{tR}, \mathrm{MS}$ \\
\hline $1720^{\mathrm{g}}$ & 1725 & Verbenone & $\mathrm{t}$ & - & 0.1 & $\mathrm{tR}, \mathrm{MS}$ \\
\hline $1708^{\mathrm{g}}$ & 1726 & Germacrene D & - & 0.6 & - & MS \\
\hline $1727^{g}$ & 1741 & $\beta$-Bisabolene & - & 0.2 & - & MS \\
\hline $1725^{\mathrm{g}}$ & 1742 & Geranial & - & 0.4 & - & $\mathrm{tR}, \mathrm{MS}$ \\
\hline $1729^{\mathrm{g}}$ & 1748 & Piperitone & - & - & 1.4 & MS \\
\hline \multirow[t]{3}{*}{$1733^{\mathrm{g}}$} & 1751 & Carvone & - & - & $\mathrm{t}$ & $\mathrm{tR}, \mathrm{MS}$ \\
\hline & 1758 & cis-Piperitol & $\mathrm{t}$ & 0.2 & 0.3 & MS \\
\hline & 1760 & Chrysanthenyl isovalerate II & - & - & 0.1 & MS \\
\hline $1762^{\mathrm{g}}$ & 1764 & cis-Chrysanthenol & 3.0 & - & - & MS \\
\hline \multirow[t]{2}{*}{$1754^{\mathrm{g}}$} & 1766 & Decanol & - & 0.5 & - & $\mathrm{tR}, \mathrm{MS}$ \\
\hline & 1770 & Isobornyl isovalerate & - & - & 0.1 & MS \\
\hline $1738^{\mathrm{g}}$ & 1770 & trans-Linalool oxide (Pyranoid) & - & 0.2 & - & MS \\
\hline \multirow[t]{2}{*}{$1773^{\mathrm{g}}$} & 1786 & ar-Curcumene & - & - & 0.2 & MS \\
\hline & 1793 & $\alpha$-Campholene alcohol & - & - & 0.2 & MS \\
\hline $1784^{\mathrm{g}}$ & 1802 & Cumin aldehyde & - & - & 0.2 & $\mathrm{tR}, \mathrm{MS}$ \\
\hline $1790^{\mathrm{h}}$ & 1804 & Myrtenol & 0.3 & 3.0 & 0.1 & MS \\
\hline $1803^{\mathrm{g}}$ & 1811 & trans- $p$-Mentha-1(7),8-dien-2-ol & - & - & 0.1 & MS \\
\hline $1836^{\mathrm{g}}$ & 1845 & trans-Carveol & 0.2 & - & 0.2 & $\mathrm{tR}, \mathrm{MS}$ \\
\hline $1839^{\mathrm{g}}$ & 1857 & Geraniol & - & 0.9 & - & $\mathrm{tR}, \mathrm{MS}$ \\
\hline \multirow[t]{4}{*}{$1848^{\mathrm{g}}$} & 1864 & $p$-Cymen-8-ol & 0.1 & 0.2 & 0.4 & $\mathrm{tR}, \mathrm{MS}$ \\
\hline & 1889 & Ascaridol & 0.4 & - & - & MS \\
\hline & 1902 & Benzyl isovalerate & - & - & 0.1 & $\mathrm{tR}, \mathrm{MS}$ \\
\hline & 1945 & 1,5-Epoxy-salvial(4)14-ene & - & 0.6 & - & MS \\
\hline \multirow[t]{2}{*}{$1955^{\mathrm{g}}$} & 1969 & cis-Jasmone & - & 0.4 & - & MS \\
\hline & 2001 & Isocaryophyllene oxide & - & - & 0.5 & MS \\
\hline $1962^{\mathrm{h}}$ & 2008 & Caryophyllene oxide & 0.1 & 0.9 & 4.7 & $\mathrm{tR}, \mathrm{MS}$ \\
\hline $2006^{\mathrm{h}}$ & 2029 & Perilla alcohol & 0.1 & 0.6 & - & MS \\
\hline \multirow[t]{3}{*}{$2006^{\mathrm{h}}$} & 2030 & Methyl eugenol & - & 1.1 & - & $\mathrm{tR}, \mathrm{MS}$ \\
\hline & 2030 & Presilphiperfolan- $9 \alpha-o l$ & - & - & 0.1 & MS \\
\hline & 2033 & $4 \alpha$-Hydroxy achipendol & 0.2 & - & - & MS \\
\hline $2016^{\mathrm{h}}$ & 2037 & Salvial-4(14)-en-1-one & - & 0.5 & 0.2 & MS \\
\hline \multirow[t]{3}{*}{$2036^{\mathrm{h}}$} & 2050 & (E)-Nerolidol & - & 2.0 & - & MS \\
\hline & 2056 & 13-Tetradecanolide & - & 0.8 & 0.6 & MS \\
\hline & 2061 & $\beta$-trans-Bejarol & - & 1.0 & - & MS \\
\hline \multirow[t]{6}{*}{$2047^{\mathrm{h}}$} & 2071 & Humulene epoxide-II & - & - & 0.4 & MS \\
\hline & 2113 & Cumin alcohol & 0.2 & - & 0.1 & $\mathrm{tR}, \mathrm{MS}$ \\
\hline & 2118 & $\alpha$-trans-Bejarol & - & 0.2 & - & MS \\
\hline & 2122 & cis-Bejarol & - & 0.5 & - & $\mathrm{tR}, \mathrm{MS}$ \\
\hline & 2130 & Salviadienol & - & 0.3 & - & MS \\
\hline & 2131 & Silphiperfol-6-en-5-one & & - & 0.7 & MS \\
\hline \multirow[t]{2}{*}{$2126^{\mathrm{h}}$} & 2144 & Spathulenol & 0.2 & 5.8 & 3.4 & MS \\
\hline & 2174 & Fokienol & - & 0.4 & - & MS \\
\hline
\end{tabular}




\begin{tabular}{|c|c|c|c|c|c|c|}
\hline & 2181 & Isothymol & - & - & 0.1 & $\mathrm{tR}, \mathrm{MS}$ \\
\hline $2176^{\mathrm{g}}$ & 2185 & $\gamma$-Eudesmol & - & 0.9 & - & MS \\
\hline \multirow[t]{2}{*}{$2165^{\mathrm{g}}$} & 2187 & T-Cadinol & - & - & 0.2 & MS \\
\hline & & Table 2 Continued & & & & \\
\hline \multirow[t]{6}{*}{$2164^{g}$} & 2198 & Thymol & - & - & 0.1 & $\mathrm{tR}, \mathrm{MS}$ \\
\hline & 2204 & Eremoligenol & - & 0.6 & - & MS \\
\hline & 2210 & Copaborneol & 0.1 & - & - & MS \\
\hline & 2214 & ar-Turmerol & - & - & 0.3 & MS \\
\hline & 2221 & Isocarvacrol & - & - & 0.3 & $\mathrm{tR}, \mathrm{MS}$ \\
\hline & 2232 & $\alpha$-Bisabolol & - & 0.3 & - & MS \\
\hline \multirow[t]{2}{*}{$2210^{\mathrm{g}}$} & 2239 & Carvacrol & 0.1 & 1.5 & 0.1 & $\mathrm{tR}, \mathrm{MS}$ \\
\hline & 2247 & trans- $\alpha$-Bergamotol & - & 0.2 & 0.2 & MS \\
\hline \multirow[t]{2}{*}{$2238^{\mathrm{h}}$} & 2257 & $\beta$-Eudesmol & 0.1 & 4.7 & - & MS \\
\hline & 2260 & 15-Hexadecanolide & - & 1.8 & 0.6 & MS \\
\hline \multirow[t]{15}{*}{$2278^{\mathrm{h}}$} & 2278 & Torilenol & - & 0.3 & - & MS \\
\hline & 2298 & Decanoic acid & - & 1.0 & 0.3 & $\mathrm{tR}, \mathrm{MS}$ \\
\hline & 2300 & Tricosane & 0.2 & - & - & $\mathrm{tR}, \mathrm{MS}$ \\
\hline & 2369 & Eudesma-4(15),7-dien-4 $\beta$-ol & - & 0.5 & 0.2 & MS \\
\hline & 2392 & $\begin{array}{l}\text { Caryophylla-2(12),6-dien-5 } \beta \text {-ol } \\
(=\text { Caryophyllenol II) }\end{array}$ & - & - & 0.6 & MS \\
\hline & 2396 & $\gamma$-Dodecalactone & - & - & 0.4 & MS \\
\hline & 2500 & Pentacosane & - & - & 0.1 & $\mathrm{tR}, \mathrm{MS}$ \\
\hline & 2533 & $\gamma$-Costol & $\mathrm{t}$ & 0.8 & - & MS \\
\hline & 2541 & Methyl nonadecanoate & - & 0.5 & - & MS \\
\hline & 2604 & $\alpha$-Costol & $\mathrm{t}$ & - & - & MS \\
\hline & 2606 & $\beta$-Costol & 0.2 & - & - & MS \\
\hline & 2607 & Octadecanol & - & 0.8 & - & MS \\
\hline & 2622 & Phytol & - & 0.2 & - & MS \\
\hline & 2700 & Heptacosane & - & $\operatorname{tr}$ & 0.2 & $\mathrm{tR}, \mathrm{MS}$ \\
\hline & & & 93.0 & 91.7 & 85.9 & \\
\hline
\end{tabular}

${ }^{\mathrm{a} R R I}$ indices from literature $\mathrm{f}$ [13], g [14], h [15], ${ }^{\mathrm{b}} \mathrm{RRI}$ : Relative retention indices calculated against $n$-alkanes; ${ }^{\mathrm{c}} \%$ : calculated from FID data; ${ }^{\mathrm{d}} \mathrm{IM}$ : Identification Method; tR, identification based on the retention times (tR) of genuine standard compounds on the HP Innowax column; MS, tentatively identified on the basis of computer matching of the mass spectra with those of the Wiley and MassFinder libraries and comparison with literature data. ${ }^{\mathrm{e}} \mathrm{t}$ : Trace $(<0.1 \%)$; Af: A. filipendulina; Am: A. magnifica; At: A. tenuifolia.

The main components of $A$. tenuifolia oil were determined as artemisia ketone (12.4\%), $p$-cymene (9.9\%), camphor (7.1\%), terpinen-4-ol (5.9\%), caryophyllene oxide $(4.7 \%)$ and $\alpha$-pinene $(4.5 \%) .85$ components were identified representing $85.9 \%$ of the total $A$. tenuifolia essential oil.

In the current study, these three Achillea essential oils were evaluated for their antimalarial and antimicrobial activities. A. magnifica and A. filipendulina oils showed strong antimalarial activity against both chloroquine-sensitive $\mathrm{D} 6\left(\mathrm{IC}_{50}=1.2\right.$ and $\left.0.68 \mu \mathrm{g} / \mathrm{mL}\right)$ and chloroquine-resistant $\mathrm{W} 2\left(\mathrm{IC}_{50}=1.1\right.$ and 0.9 $\mu \mathrm{g} / \mathrm{mL})$ strains of Plasmodium falciparum when the comported to positive standards chloroquine $\left(\mathrm{IC}_{50}=\right.$ $0.018 \mu \mathrm{g} / \mathrm{mL}$ for D6 and $\mathrm{IC}_{50}=0.16 \mu \mathrm{g} / \mathrm{mL}$ for $\mathrm{W} 2$ clones $)$ and artemisinin $\left(\mathrm{IC}_{50}=0.0037 \mu \mathrm{g} / \mathrm{mL}\right.$ for D6 and $\mathrm{IC}_{50}=0.0035 \mu \mathrm{g} / \mathrm{mL}$ for $\mathrm{W} 2$ clones) without exhibiting any cytotoxicity at $\mathrm{IC}_{50}$ values of $47.6,15.867$ and $5.288 \mu \mathrm{g} / \mathrm{mL}$ against Vero cells. however, A. tenuifolia did not show antimalarial activity Achillea oils showed no antibacterial activity against human pathogenic bacteria up to a concentration of $200 \mu \mathrm{g} / \mathrm{mL}$. $A$. tenuifolia and A. magnifica oils demonstrated mild antifungal activity against Cryptococcus neoformans $\left(\mathrm{IC}_{50}=20\right.$ and $15 \mu \mathrm{g} / \mathrm{mL}$, respectively) and A. tenuifolia oil demonstrated weak antimycobacterial activity against Mycobacterium intracellulare with an $\mathrm{IC}_{50}$ value of $200 \mu \mathrm{g} / \mathrm{mL}$. Bioassay-guided investigations are warranted out to identify active antimalarial compounds from A. magnifica and A. filipendulina essential oils.

To the best of our knowledge, this is the first report of chemical composition these three Achillea species from eastern (Malatya and Ağrı) and south-eastern (Şırnak) region of Turkey and their biological activities of $A$. magnifica, A. tenuifolia and A. filipendulina essential oils were evaluated. 


\section{Acknowledgments}

The authors thank Mr. John Trott, Ms. Marsha Wright for excellent technical support in performing antimalarial and antimicrobial assays. This work was supported in part by the NIH, NIAID, Division of AIDS, Grant No. AI 27094 (antifungal) and the USDA Agricultural Research Service Specific Cooperative Agreement No. 58-6408-1-603.

\section{ORCID}

Betül Demirci: 0000-0003-2343-746X

K. Hüsnü Can Başer: 0000-0003-2710-0231

Zeki Aytaç: 0000-0003-3244-3183

Shabana I. Khan: 0000-0001-6429-7219

Melissa R. Jacob: 0000-0003-4478-3345

Nurhayat Tabanca: 0000-0003-2802-8796

\section{References}

[1] N. Tabanca, B. Demirci, Z. Aytac and K. H. C. Baser (2016). Chemical composition of Achillea schischkinii Sosn., an endemic species from Turkey, Nat. Volatiles Essent. Oils. 3, 24-28.

[2] K. H. C. Baser (2016). Essential oils of Achillea species of Turkey, Nat. Volatiles Essent. Oils 3, 1-14.

[3] K. H. C. Baser, B. Demirci and H. Duman (2001). Composition of the essential oils of two endemic species from Turkey: Achillea lycaonica and A. ketenoglui, Chem. Nat. Compd. 37, 245-252.

[4] E. Tuzlacı (2006). Türkiye'nin Bitkisel Halk Illaçları, Alfa Basım Yayım Dağıtım Şirketi, Istanbul, 17pp.

[5] M. Mohammadhosseini, S. D. Sarker and A. Akbarzadeh (2017). Chemical composition of the essential oils and extract of Achillea species and their biological activities: A review, J. Ethnopharmacol. 199, 257-315.

[6] A. Ebadollahi (2017). Chemical composition, acaricidal and insecticidal effects of essential oil from Achillea filipendulina against two arthropod pests; Oryzaephilus surinamensis and Tetranychus urticae, Toxin Reviews 36, 132-137.

[7] M. Piryaei and H. Nazemiyeh (2016). Fast analysis of volatile components of Achillea tenuifolia Lam with microwave distillation followed by headspace single-drop microextraction coupled to gas chromatographymass spectrometry (GC-MS), Nat. Prod. Res. 30, 991-994.

[8] S. Gharibi, B. E. S. Tabatabaei and G. Saeidi (2015). Comparison of essential oil composition, flavonoid content and antioxidant activity in eight Achillea species, J. Essent. Oil-Bear. Plants 18, 1382-1394.

[9] K. Mohsen (2015). Chemical composition of the essential oil of Achillea tenufolia aerial parts, J. Essent. OilBear. Plants 18, 261-263.

[10] O. Toncer, S. Basbag, S. Karaman, E. Diraz, M. Basbag (2010). Chemical composition of the essential oils of some Achillea Species growing wild in Turkey, Int. J. Agric. Biol. 12, 527-530.

[11] N. Tabanca, E. Bedir, N. Kırımer, K.H.C. Baser, S. I. Khan, M. R. Jacob, I. A. Khan (2003). Antimicrobial compounds from Pimpinella species growing in Turkey, Planta Med. 69, 933-938.

[12] N. Tabanca, E. Bedir, D. Ferraira, D. Slade, D. E. Wedge, M. R. Jacob, S. I. Khan, N. Kırımer, K. H. C. Baser and I. A. Khan (2005). Bioactive constituents from Turkish Pimpinella species, Chem. Biodiv. 2, 221-232.

[13] N. Tan, S. Yazıc1-Tütüniş, Y. Yeşil, B. Demirci and E. Tan (2017). Antibacterial activities and composition of the essential oils of Salvia sericeo-tomentosa varieties, Rec. Nat. Prod. 11, 456-461.

[14] V. I. Babushok, P.J. Linstrom and I.G. Zenkevich (2011). Retention indices for frequently reported compounds of plant essential oils, J. Phys. Chem. Ref. Data, 40(4), doi:10.1063/1.3653552

[15] H. E. Temel, B. Demirci, F. Demirci, F. Celep, A. Kahraman, M. Doğan and K. H. C. Başer (2016). Chemical characterization and anticholinesterase effects of essential oils derived from Salvia species, J. Essent. Oil Res. 28, 322-331.

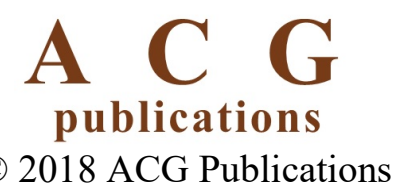

